\title{
Neural precursor cell expressed, developmentally downregulated 8-activating enzyme inhibitor MLN4924 sensitizes colorectal cancer cells to oxaliplatin by inducing DNA damage, $\mathbf{G} 2$ cell cycle arrest and apoptosis
}

\author{
WANWEI ZHENG ${ }^{1}{ }^{*}$, ZHONGGUANG LUO $^{1 *}$, JUN ZHANG $^{1}$, PEI MIN ${ }^{2}$, WENSHUAI LI ${ }^{1}$, \\ DIANNAN XU ${ }^{1}$, ZIQIANG ZHANG ${ }^{3}$, PANPAN XIONG ${ }^{4}$, HONG LIANG ${ }^{1}$ and JIE LIU ${ }^{1,5}$ \\ ${ }^{1}$ Department of Digestive Diseases, Huashan Hospital, Fudan University, Shanghai 200040; \\ ${ }^{2}$ Department of Digestive Diseases, Southeast Hospital, Xiamen University, Zhangzhou, Fujian 363000; \\ ${ }^{3}$ Department of General Surgery, Huashan Hospital, Fudan University, Shanghai 200040; ${ }^{4}$ Department of \\ Digestive Diseases, East Hospital, Tongji University, Shanghai 200120; ${ }^{5}$ Department of Immunology, \\ Institute of Biomedical Sciences, Shanghai Medical School, Fudan University, Shanghai 200032, P.R. China
}

Received January 13, 2016; Accepted January 19, 2017

DOI: $10.3892 / \mathrm{mmr} .2017 .6305$

\begin{abstract}
Oxaliplatin-based chemotherapy is a primary treatment for patients with metastatic colorectal cancer (CRC); however, its efficacy is limited. Therefore, novel therapeutic agents are urgently required. MLN4924 is a first-in-class inhibitor of neural precursor cell expressed, developmentally downregulated 8 (NEDD8)-activating enzyme E1, and has entered various phase-I/II clinical trials for cancer therapy due to its significant anticancer efficacy. The aim of the present study was to examine the synergistic effect and underlying mechanisms of MLN4924 and oxaliplatin combined treatment for CRC. It was demonstrated that MLN4924 treatment induced the DNA damage response (DDR) by inactivating cullin-ring ubiquitin ligases, subsequently leading to cell cycle disturbance and apoptosis in CRC cells. MLN4924 treatment increased the oxaliplatin-induced DDR, G2 cell cycle arrest and apoptosis. Protein expression levels of phosphorylated checkpoint kinase 2 (p-CHK2), p21 and p53, which are well-known functional proteins involved in G2 cell cycle arrest, were assessed. p-CHK2 protein expression levels were increased following combined treatment with MLN4924 and oxaliplatin, whereas $\mathrm{p} 21 / \mathrm{p} 53$ protein expression levels were not. In conclusion, MLN4924 treatment may sensitize CRC cells to oxaliplatin treatment by inducing the DDR and increasing
\end{abstract}

Correspondence to: Dr Jie Liu, Department of Digestive Diseases, Huashan Hospital, Fudan University, 12 Middle Wulumuqi Road, Shanghai 200040, P.R. China

E-mail: jieliu@fudan.edu.cn

*Contributed equally

Key words: MLN4924, oxaliplatin, DNA damage response, G2 cell cycle arrest, apoptosis, colorectal cancer protein expression levels of p-CHK2, leading to G2 cell cycle arrest and apoptosis. Therefore, combined MLN4924 and oxaliplatin-based chemotherapy may be a potential therapeutic strategy for the treatment of CRC.

\section{Introduction}

Colorectal cancer (CRC) is currently one of the most commonly diagnosed types of cancer worldwide, with an estimated 1.4 million cases leading to 693,900 fatalities in 2012 (1). More than half of patients (50-60\%) diagnosed with CRC develop metastases $(2,3)$.

Oxaliplatin is a third-generation platinum analog that may disrupt DNA replication and transcription, leading to DNA damage and cell apoptosis $(4,5)$. Oxaliplatin-based chemotherapy is a primary treatment for patients with metastatic CRC; however, its efficacy is limited. The combination of oxaliplatin-based chemotherapy and targeted therapy, including bevacizumab, panitumumab and cetuximab, may prolong progression-free survival by only 1-3 months (6-8). Novel therapeutic agents are urgently required to improve survival rates.

The cullin-ring ubiquitin ligases (CRL), a subset of E3 ligases (9) and the predominant multi-unit ubiquitin ligase family in cells, are involved in the degradation of $20 \%$ of ubiquitinated cellular proteins to regulate a wide variety of biologic processes $(10,11)$. MLN4924 is a first-in-class inhibitor of neural precursor cell expressed, developmentally downregulated 8 (NEDD8)-activating enzyme E1 that was discovered via high-throughput screening $(10,12)$, and which has entered various phase-I/II clinical trials for cancer therapy due to its significant anticancer efficacy in preclinical studies (13). Via the formation of an inactive covalent NEDD8-MLN4924 adduct (14), MLN4924 blocks CRL neddylation, leading to accumulation of a mass of CRL substrates $(10,15,16)$, and resulting in DNA re-replication, the DNA damage response 
(DDR), abnormal cell cycle progression, apoptosis, autophagy and senescence, thus inhibiting the growth of cancer cells $(10,17-22)$.

MLN4924 and oxaliplatin are anticancer agents that may induce the DDR. There is potentially a synergistic effect between MLN4924 and oxaliplatin in inducing the DDR, leading to cell cycle arrest and apoptosis, and resulting in improved anticancer efficacy. The aim of the present study was to examine the synergistic effect and specific underlying mechanisms of MLN4924 and oxaliplatin combined treatment of CRC.

\section{Materials and methods}

Cell lines, culture and reagents. SW620 and HCT116 human colorectal carcinoma cell lines were obtained from the American Type Culture Collection (Manassas, VA, USA), and cultured in RPMI-1640 (Gibco; Thermo Fisher Scientific, Inc., Waltham, MA, USA) supplemented with $10 \%$ fetal bovine serum (Biochrom, Ltd., Cambridge, UK) and $1 \%$ penicillin-streptomycin solution, at $37^{\circ} \mathrm{C}$ in $5 \% \mathrm{CO}_{2}$. MLN4924 (Takeda Pharmaceutical Co., Ltd., Tokyo, Japan) was dissolved in dimethyl sulfoxide (DMSO) and stored at $-20^{\circ} \mathrm{C}$. MLN4924 solution was freshly made every week and stored in the dark at room temperature prior to use. Oxaliplatin (Sigma-Aldrich; Merck Millipore, Darmstadt, Germany) was dissolved in sterile water and stored at $-20^{\circ} \mathrm{C}$. SW620 and HCT116 were seeded into 24 -well plates at a density of $1 \times 10^{4}$ cells/well or 12 -well at a density of $2 \times 10^{5}$ cells/well, and treated with $0.1,0.3$ or $1.0 \mu \mathrm{mol} / 1 \mathrm{MLN} 4924,0.3 \mu \mathrm{mol} / 1$ oxaliplatin, or $0.3 \mu \mathrm{mol} / 1$ MLN4924 combined with $0.3 \mu \mathrm{mol} / 1$ oxaliplatin for $24,48,72$ or $96 \mathrm{~h}$ at $37^{\circ} \mathrm{C}$.

Western blotting. Cells were lysed with radioimmunoprecipitation assay lysis buffer (Beyotime Institute of Biotechnology, Haimen, China) containing phenylmethylsulfonyl fluoride (Beyotime Institute of Biotechnology), and lysates were centrifuged at $13,500 \times g$ for $5 \mathrm{~min}$ at $4^{\circ} \mathrm{C}$. Protein concentrations were quantified using a Bicinchoninic Acid kit (Thermo Fisher Scientific, Inc.). A total of $30 \mu \mathrm{g}$ protein was separated using $10 \%$ SDS-PAGE gels, and transferred to polyvinylidene difluoride membranes. The membranes were blocked with $5 \%$ non-fat milk in TBS containing Tween-20 (TBST) for $1 \mathrm{~h}$ at room temperature, and subsequently incubated with the following primary antibodies overnight at $4^{\circ} \mathrm{C}$ : Mouse anti-cullin 1 (1:1,000; catalog no. sc-17775; Santa Cruz Biotechnology, Inc., Dallas, TX, USA), rabbit anti-p21 (1:1,000; catalog no. 3733-1; Epitomics, Burlingame, CA, USA), rabbit anti-total checkpoint kinase 2 (t-CHK2; 1:1,000; catalog no. 3428-1; Epitomics), rabbit anti-total histone $\mathrm{H} 2 \mathrm{~A}(\mathrm{t}-\mathrm{H} 2 \mathrm{~A} ; 1: 1,000$; catalog no. 3522-1; Epitomics), rabbit anti-phosphorylated (p)-CHK2 (Thr68; 1:1,000; catalog no. 2197; Cell Signaling Technology, Inc., Danvers, MA, USA), rabbit anti-p-H2A (Ser139; 1:1,000; catalog no. 2577; Cell Signaling Technology, Inc.), rabbit anti-p27 (1:1,000; catalog no. 3686; Cell Signaling Technology, Inc.), rabbit anti-p53 (1:1,000; catalog no. 2527; Cell Signaling Technology, Inc.), rabbit anti-cleaved poly adenosine diphosphate ribose polymerase (PARP; 1:1,000; catalog no. 9532; Cell Signaling Technology, Inc.) and rabbit anti- $\beta$-actin $(1: 1,000$; catalog no. ab8227; Abcam, Cambridge, UK). Subsequently, membranes were washed twice with TBST and incubated with a horseradish peroxidase (HRP)-conjugated goat anti-rabbit secondary antibody (1:2,000; catalog no. SA00001-2; Wuhan Sanying Biotechnology, Wuhan, China) or an HRP-conjugated anti-mouse secondary antibody (1:2,000; catalog no. ab131368; Abcam) for $1 \mathrm{~h}$ at room temperature. Protein bands were visualized using an Immobilon Western Chemiluminescent HRP Substrate (EMD Millipore, Billerica, MA, USA) and imaged using a Tanon 4200 Chemiluminescent Imaging system (Tanon Science and Technology Co., Ltd., Shanghai, China).

Cell counting and clonogenic assay. For cell counting, cells were seeded into 24-well plates at a density of $1 \times 10^{4}$ cells per well and treated with $0.1 \mu \mathrm{mol} / 1 \mathrm{MLN} 4924$. Cells were trypsinized, resuspended and counted using a Cellometer Auto T4 Cell Viability Counter (Nexcelom Bioscience, Lawrence, MA, USA) at $0,48,72$ and $96 \mathrm{~h}$ after MLN4924 treatment. For the clonogenic assay, cells were seeded into a $60-\mathrm{mm}$ dish at a density of 500 cells/well and cultured for 10 days after $0.1 \mu \mathrm{mol} / 1 \mathrm{MLN} 4924$ treatment. Colonies on the dish were fixed with $4 \%$ paraformaldehyde and stained with crystal violet. Colonies with $>50$ cells were counted.

Flow cytometric analysis. Cells were seeded into 6-well plates at a density of $2 \times 10^{5}$ per well and treated with DMSO, MLN4924 or oxaliplatin were harvested and fixed in $70 \%$ ethanol at $-20^{\circ} \mathrm{C}$ overnight, and stained with $36 \mathrm{mg} / \mathrm{ml}$ propidium iodide (PI; Sigma-Aldrich; Merck Millipore) containing $10 \mathrm{mg} / \mathrm{ml}$ RNase (Sigma-Aldrich; Merck Millipore) at $37^{\circ} \mathrm{C}$ for $15 \mathrm{~min}$, following which they were analyzed for apoptosis and cell cycle progression using a CyAn ${ }^{\mathrm{TM}}$ ADP analyzer (Beckman Coulter, Inc., Brea, CA, USA). Apoptosis was measured as the percentage of cells in the sub-G1 population. Data were analyzed using ModFit LT software version 4.0 (Verity Software House, Inc., Topsham, ME, USA).

Live-dead cell staining assay. A Live-Dead Cell Staining kit (Enzo Life Sciences, Inc., Farmingdale, NY, USA) was utilized to stain cells to discriminate between live and dead cells. Cells were seeded into 12 -well plates at a density of $2 \times 10^{5}$ cells per well. Solutions A (Live-Dye, a cell-permeable green fluorescent dye) and B (PI, a cell non-permeable red fluorescent dye) were mixed in solution buffer. Cells were washed once with PBS and stained with mixed staining solution at $37^{\circ} \mathrm{C}$ for $15 \mathrm{~min}$. Cells were immediately observed under a fluorescence microscope using a band-pass filter. Healthy cells were stained green and dead cells were stained red.

Statistical analysis. Data are expressed as the mean \pm standard deviation. Data were analyzed using GraphPad Prism software version 6 (GraphPad Software, Inc., La Jolla, CA, USA). An unpaired two-tailed Student's t-test was used for the comparison of parameters between groups. Multiple groups were compared using analysis of variance followed by the Bonferroni post hoc test. $\mathrm{P}<0.05$ was considered to indicate a statistically significant difference.

\section{Results}

MLN4924 inhibits cullin neddylation and growth of CRC cells. The efficacy of MLN4924 treatment on CRC cells 
A

SW620

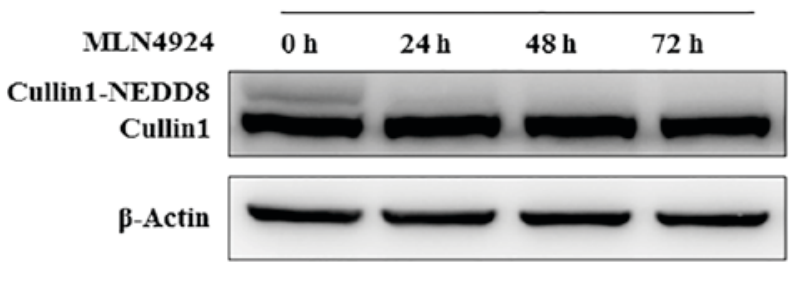

sW620

B

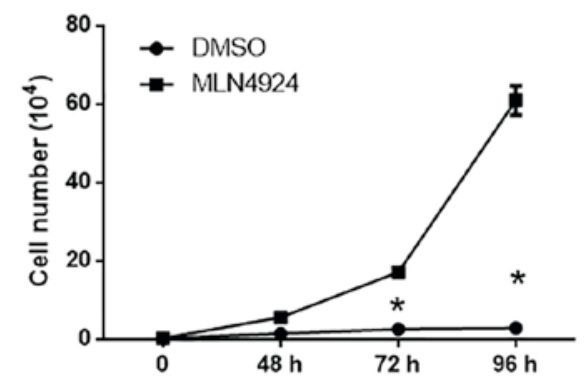

DMSO

MLN4924

C
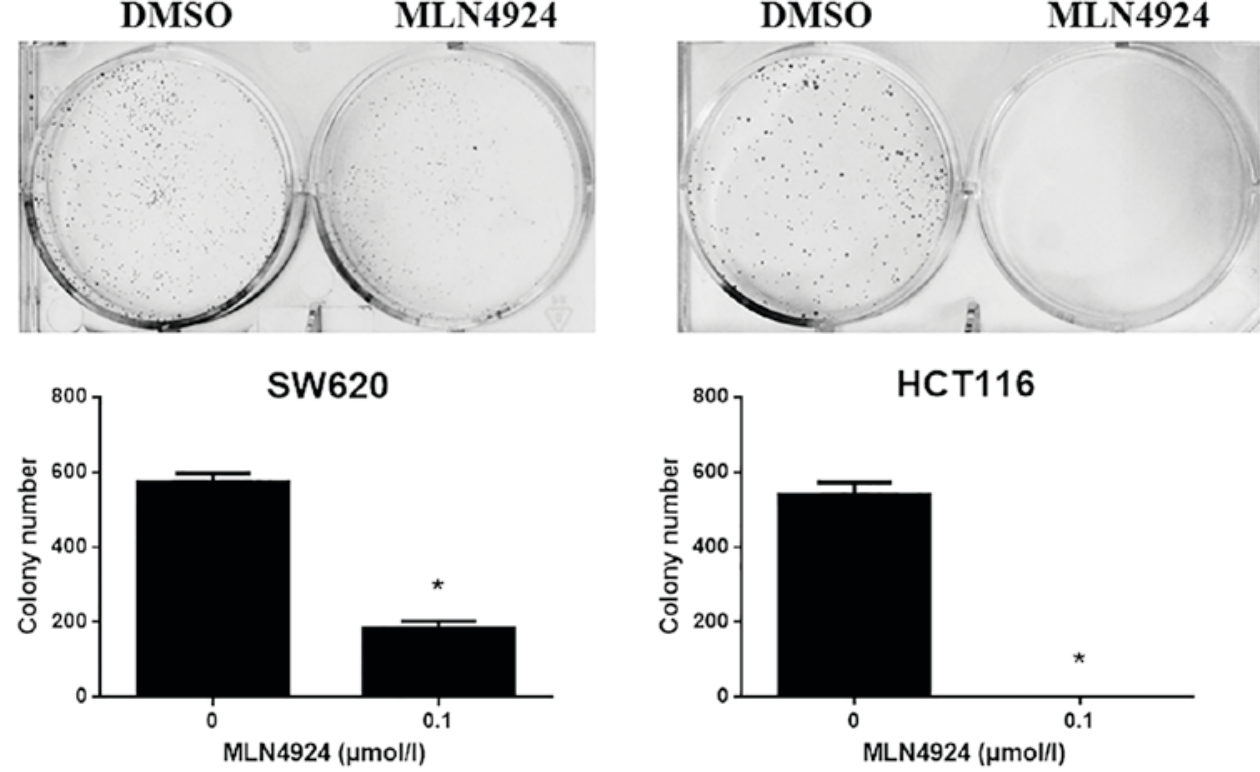

Figure 1. MLN4924 inhibits the growth of CRC cells. (A) MLN4924 treatment inhibited cullin1 neddylation, as assessed by western blot analysis; $\beta$-actin served as a loading control. (B) MLN4924 treatment inhibited CRC cell proliferation. "P<0.001 vs. DMSO treatment at the same time point. (C) MLN4924 treatment suppressed colony formation in liver cancer cells, as examined by crystal violet staining and colony counting. "P<0.001 vs. $0 \mu \mathrm{mol} / 1 \mathrm{MLN} 4924$. Data are expressed as the mean \pm standard deviation $(\mathrm{n}=3)$. CRC, colorectal cancer; NEDD-8, neural precursor cell expressed, developmentally downregulated 8 ; DMSO, dimethyl sulfoxide.

was examined. SW620 and HCT116 cells were treated with MLN4924 and subjected to western blotting and cell growth analysis. MLN4924 completely inhibited cullin1-NEDD8 protein expression levels in SW620 and HCT116 cells (Fig. 1A) and significantly suppressed the proliferation of SW620 and HCT116 cells (Fig. 1B; P<0.001). MLN4924 additionally markedly suppressed cell clonogenic survival in these cells (Fig. 1C; $\mathrm{P}<0.001$ ). These results suggested that MLN4924 significantly inhibited cullin1 neddylation and the growth of CRC cells.

MLN4924 induces the DDR, G2 cell cycle arrest and apoptosis in CRC cells. Protein expression levels of p-H2A and p-CHK2, well-known markers of the DDR, were increased as MLN4924 concentrations increased in SW620 and HCT116 cells. Additionally, p53, p21 and p27 protein expression levels increased as MLN4924 concentrations increased in the two cell lines. p21 and p27 are classical CRL substrates and p21 and p53 are involved in G2 cell cycle arrest. Protein expression levels of cleaved-PARP, an indicator of cell apoptosis, increased in a dose-dependent manner (Fig. 2A). Cell cycle analysis demonstrated that MLN4924 treatment triggered sub G1 (an indicator of apoptosis) and G2 cell cycle arrest in CRC cells (Fig. 2B and $\mathrm{C} ; \mathrm{P}<0.001$ ). These findings suggested that MLN4924 treatment induced the DDR by inactivating CRL, leading to cell cycle disturbance and apoptosis, which was consistent with previous studies (20-23).

MLN4924 increases the oxaliplatin-induced DDR and G2 cell cycle arrest. It has previously been reported that oxaliplatin is involved in DNA damage by inducing DNA 
A

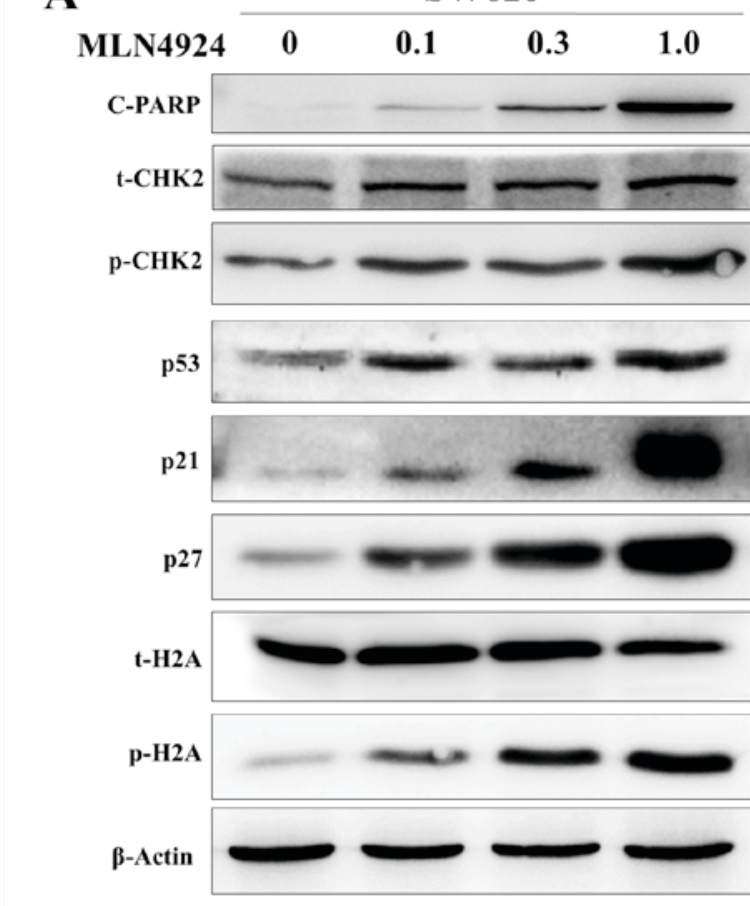

HCT116

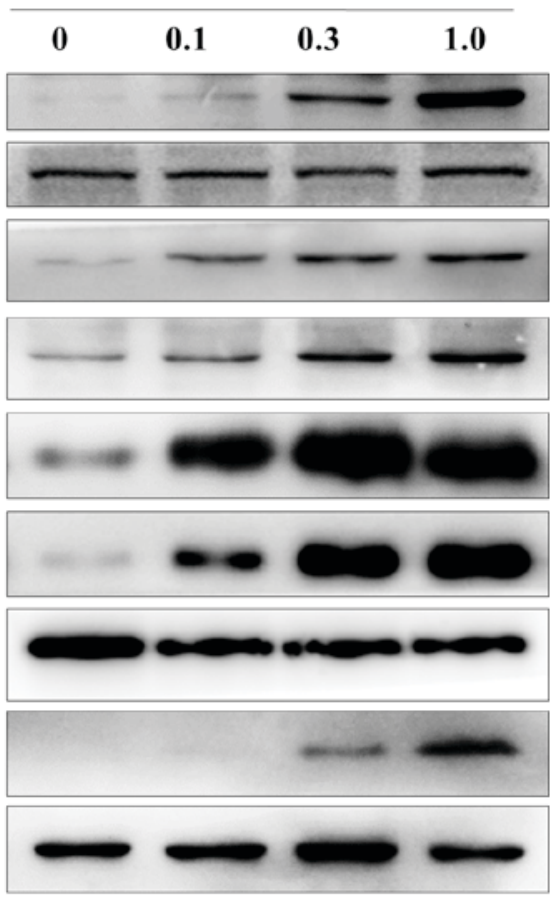

$72 \mathrm{~h}$

C
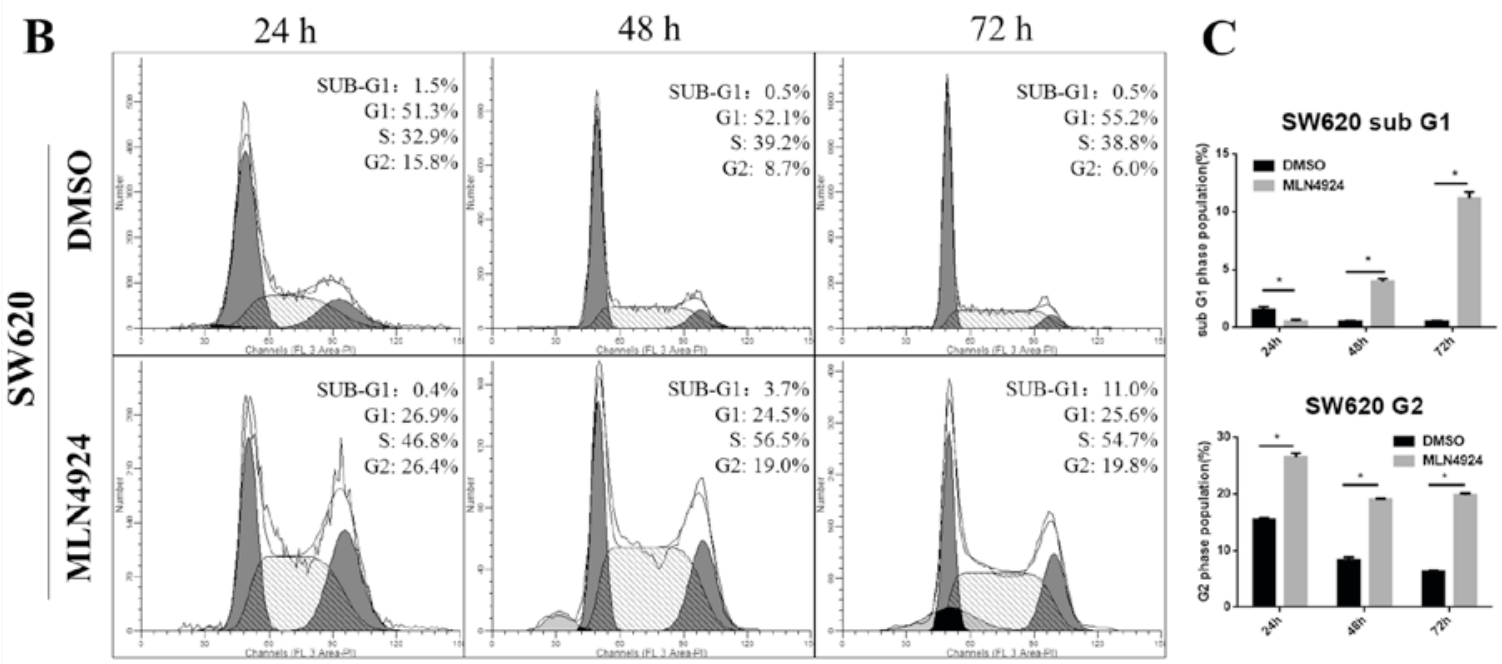

Figure 2. MLN4924 treatment induces G2 cell cycle arrest and apoptosis in colorectal cancer cells. (A) The protein expression levels of c-PARP, p-CHK2, p53, p21, p27 and p-H2A were assessed in MLN4924-treated SW620 and HCT116 cells by western blot analysis; $\beta$-actin served as a loading control. (B) MLN4924 induced cell-cycle arrest and apoptosis, as examined by propidium iodide staining and flow cytometric analysis. (C) The percentage of cells at the G2 and sub-G1 phases. Data are expressed as the mean \pm standard deviation. "P<0.001. c-PARP, cleaved poly adenosine diphosphate ribose polymerase; CHK2, checkpoint kinase 2; p, phosphorylated; H2A, histone H2A; DMSO, dimethyl sulfoxide.

cross-links and activating the DDR $(4,5)$. The present study demonstrated that MLN4924 and oxaliplatin treatment induced increased protein expression levels of p-H2A and p-CHK2 compared with single agent treatment in CRC cells. However, MLN4924 treatment in combination with oxaliplatin did not increase protein expression levels of p21 and p53 compared with single agent treatment (Fig. 3A). These results suggested that MLN4924 treatment increased the oxaliplatin-induced DDR.

Cell cycle analysis indicated that oxaliplatin treatment induced G2 cell cycle arrest in CRC cells (Fig. 3B). Cells treated with MLN4924 and oxaliplatin had an increased proportion of cells in $\mathrm{G} 2$ phase compared with cells treated with a single agent (Fig. 3B). These findings demonstrated that MLN4924 treatment increased oxaliplatin-induced G2 cell cycle arrest, accompanied by an additional increase in the sub-G1 population (Fig. 3C; P<0.001); however, p21 and p53 were not active in this process.

MLN4924 increases oxaliplatin-induced apoptosis. MLN4924 and oxaliplatin treatment increased the protein expression levels of cleaved-PARP compared with single agent treatment (Fig. 4A). Cell apoptosis was analyzed using the Live-Dead Cell Staining kit. CRC cells treated with combined MLN4924 and oxaliplatin exhibited increased apoptotic cells (stained red) compared with MLN4924 or oxaliplatin only treatment 
A

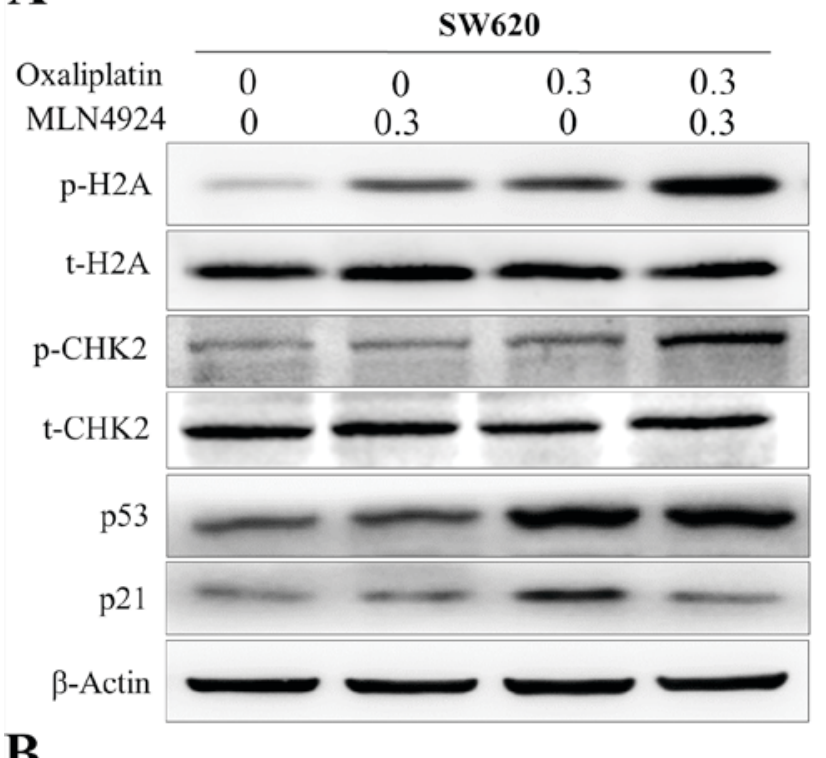

B
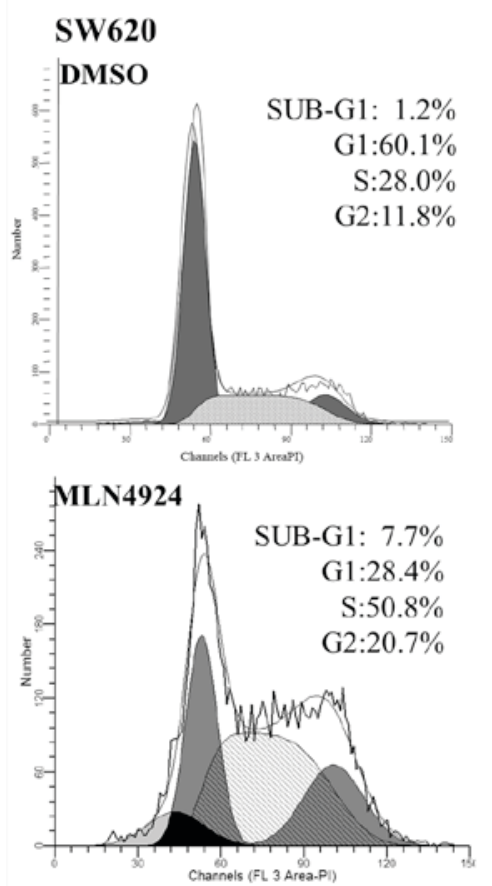
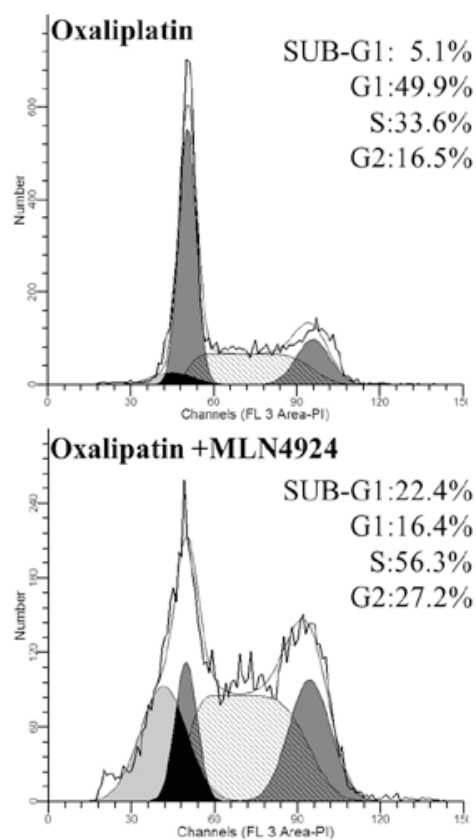

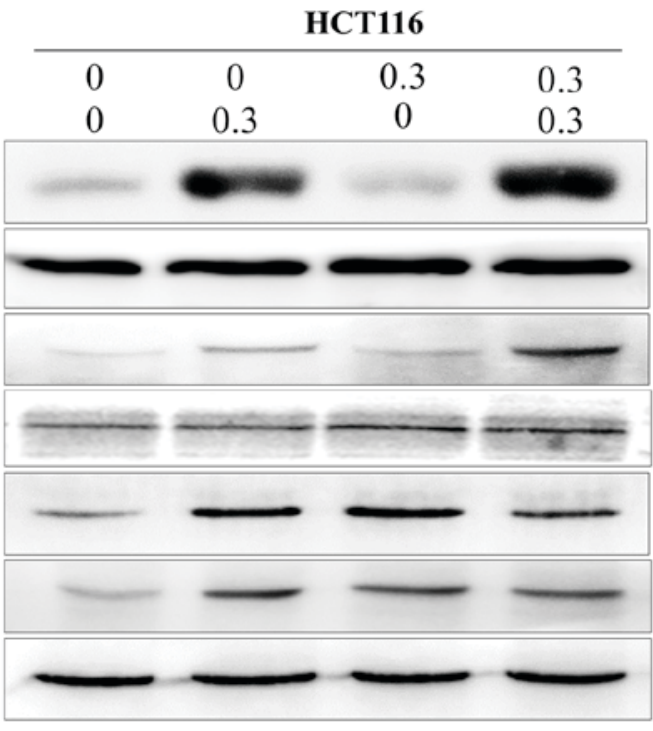

C
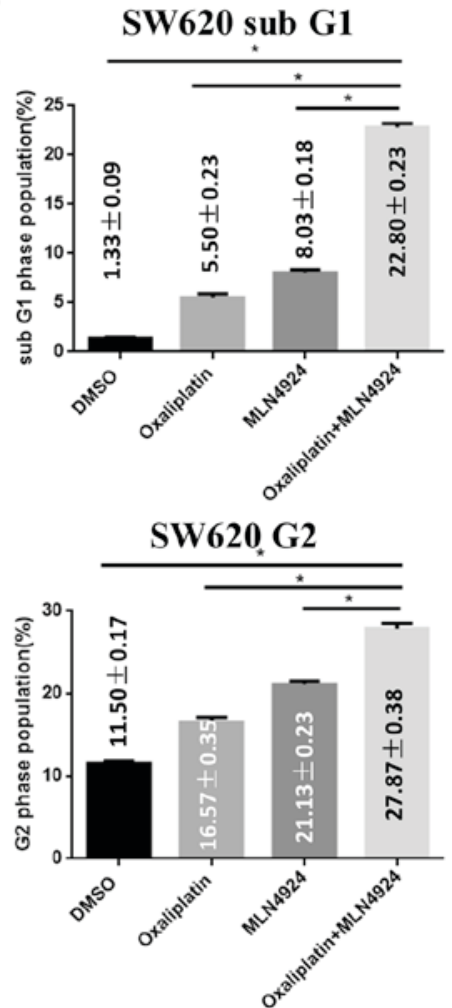

Figure 3. MLN4924 increases the oxaliplatin-induced DNA damage response, G2 cell cycle arrest and apoptosis. (A) Western blot analysis of protein expression levels of p-H2A, t-H2A, p-CHK2, t-CHK2, p53 and p21 in SW620 and HTC116 cells following single or combined MLN4924 and oxaliplatin treatment; $\beta$-actin served as a loading control. (B) Flow cytometric analysis of the cell cycle of SW620 cells following single or combined oxaliplatin and MLN4924 treatment. (C) Quantification of the percentage of cells in $\mathrm{G} 2$ and sub-G1 phases. Data are expressed as the mean \pm standard deviation. ${ }^{*} \mathrm{P}<0.001$. H2A, histone H2A; CHK2, checkpoint kinase 2; p, phosphorylated; t, total; DMSO, dimethyl sulfoxide.

(Fig. 4B). These results indicated that MLN4924 increased oxaliplatin-induced apoptosis.

\section{Discussion}

Over half of patients (50-60\%) diagnosed with CRC develop metastases $(2,3)$. Oxaliplatin-based chemotherapy is the first line of treatment for patients with metastatic CRC; however, its efficacy is limited. The combination of oxaliplatin-based chemotherapy with anti-vascular endothelial growth factor or anti-epidermal growth factor receptor agents prolongs progression-free survival by only 1-3 months (6-8). Novel therapeutic agents are urgently required to improve the anticancer efficacy of oxaliplatin.

Our previous study demonstrated that MLN4924 may induce the DDR in liver cancer cells in vivo and in vitro (23). Other previous studies additionally reported that MLN4924 may induce the DDR and apoptosis in multiple cancer cell 
A

\begin{tabular}{ccccc}
\cline { 2 - 5 } MLN4924 & 0 & 0.3 & 0 & 0.3 \\
Oxaliplatin & 0 & 0 & 0.3 & 0.3 \\
\cline { 3 - 5 } & & & & \\
C-PARP
\end{tabular}

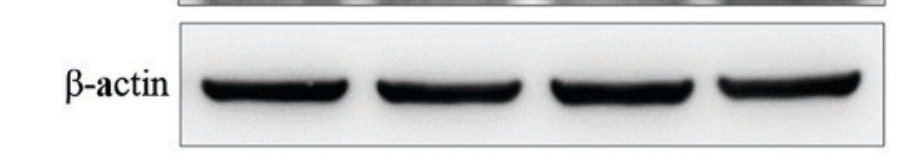

B

\begin{tabular}{llccc}
\cline { 2 - 3 } ML924 & 0 & 0.3 & 0 & 0.3 \\
Oxaliplatin & 0 & 0 & 0.3 & 0.3
\end{tabular}
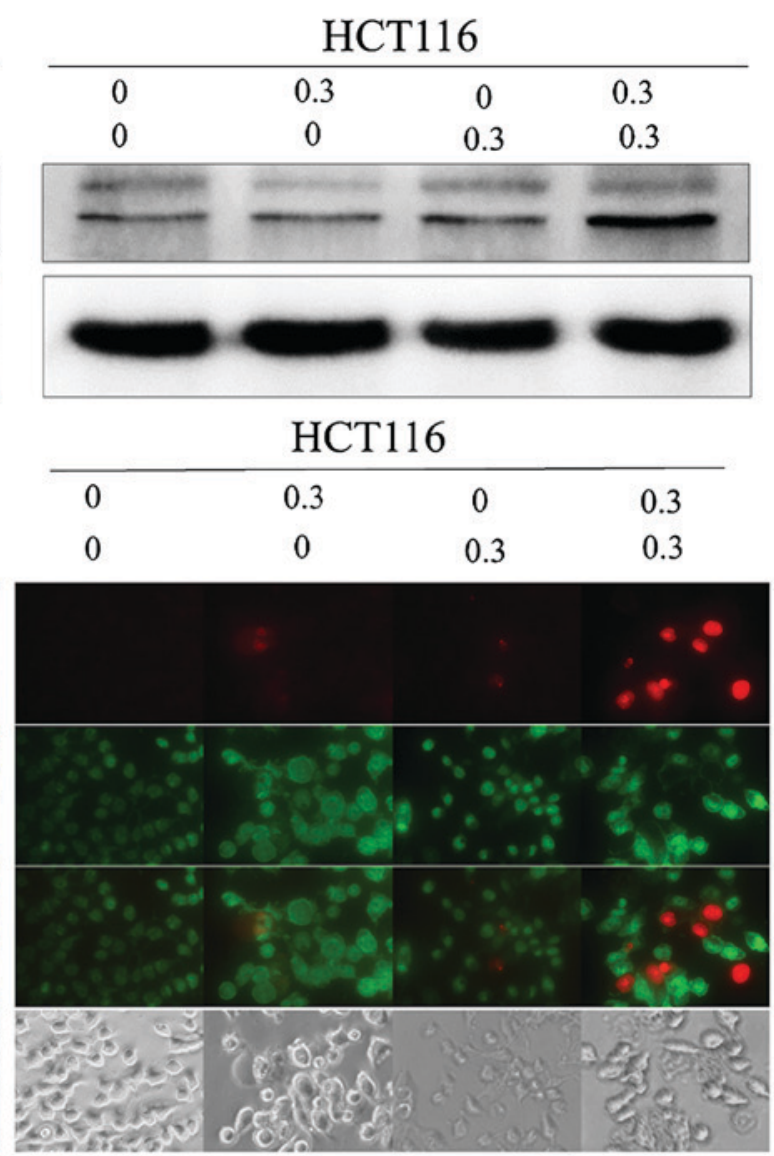

Figure 4. MLN4924 treatment increases oxaliplatin-induced apoptosis. (A) Western blot analysis of c-PARP protein expression levels in SW620 and HCT116 cells following MLN4924 and oxaliplatin treatment; $\beta$-actin served as a loading control. (B) Cells were stained using a Live-Dead Cell Staining kit and observed under a fluorescence microscope. Healthy cells are stained green and dead cells are stained red. Original magnification, $\mathrm{x} 400$. c-PARP, cleaved poly adenosine diphosphate ribose polymerase.

lines $(17,18)$. The present study hypothesized that MLN4924 treatment may induce the DDR in CRC cells, which may sensitize these cells to oxaliplatin. MLN4924 treatment was demonstrated to enhance the anticancer efficacy of oxaliplatin in CRC cells by increasing oxaliplatin-induced DDR, G2 cell cycle arrest and apoptosis.

Oxaliplatin forms inter- and intra-strand cross-links with DNA, leading to the DDR (24). MLN4924 additionally induces the DDR by accumulation of chromatin licensing and DNA replication factor 1 (21), inhibition of the nuclear factor- $\kappa \mathrm{B}$ signaling pathway (19) and increase of reactive oxygen species (ROS) generation (25). p-H2A expression levels are increased according to the DNA damage level, and are thus used as a typical maker of the DDR (26). CHK2 is phosphorylated in response to DNA damage, is involved in the DNA damage signaling pathway, and is additionally used as a maker of the DDR (27). The present study demonstrated that combined MLN4924 and oxaliplatin treatment induced increased protein expression levels of $\mathrm{p}-\mathrm{H} 2 \mathrm{~A}$ and $\mathrm{p}-\mathrm{CHK} 2$ compared with single treatment. These results indicated that MLN4924 may significantly increase oxaliplatin-induced DDR.

Furthermore, MLN4924 treatment was revealed to increase oxaliplatin-induced G2 cell cycle arrest. p21 and p53 are two well-known functional proteins of this cell cycle disturbance process $(28,29)$. The present study demonstrated that MLN4924 or oxaliplatin treatment increased the protein expression levels of p21 and p53 in CRC cells; however, combined treatment did not increase protein expression levels of p21 and p53 further. These results suggested that p21 and p53 were not active in the process by which MLN4924 increased oxaliplatin-induced G2 cell cycle arrest. DNA damage may activate checkpoints, and phosphorylation of CHK2 is widely reported to lead to G2 cell cycle arrest $(27,30)$. The present study demonstrated that p-CHK2 protein expression levels were increased following combined treatment, indicating that it may have mediated the G2 cell cycle arrest process.

Previous studies have reported that MLN4924 treatment increases the cisplatin-induced DDR and apoptosis in multiple cell lines, via inhibiting monoubiquitination of fanconi anemia group D2 (31) and increasing ROS generation (25). Oxaliplatin is a third-generation platinum analog, and is more effective in the treatment of CRC compared with cisplatin (32). The specific mechanisms underlying the increase of the oxaliplatin-induced DDR and G2 cell cycle arrest in CRC cells by MLN4924 require further investigation.

In conclusion, the present study revealed that the NEDD8-activating enzyme inhibitor MLN4924 sensitizes CRC cells to oxaliplatin by inducing the DDR and increasing protein expression levels of p-CHK2, leading to $\mathrm{G} 2$ cell cycle arrest and apoptosis. These findings provide evidence for the potential efficacy of combined MLN4924- and oxaliplatin-based chemotherapy for the treatment of CRC. 


\section{Acknowledgements}

The present study was supported by the Shanghai Municipal Commission of Health and Family Planning (grant no. 20144Y0094) and the National Natural Science Foundation of China (grant nos. 81500503 and 81420108005).

\section{References}

1. Torre LA, Bray F, Siegel RL, Ferlay J, Lortet-Tieulent J and Jemal A: Global cancer statistics, 2012. CA Cancer J Clin 65: 87-108, 2015.

2. Lee WS, Yun SH, Chun HK, Lee WY, Yun HR, Kim J, Kim K and Shim YM: Pulmonary resection for metastases from colorectal cancer: Prognostic factors and survival. Int J Colorectal Dis 22: 699-704, 2007

3. Yoo PS, Lopez-Soler RI, Longo WE and Cha CH: Liver resection for metastatic colorectal cancer in the age of neoadjuvant chemotherapy and bevacizumab. Clin Colorectal Cancer 6: 202-207, 2006

4. Mowaka S, Ziehe M, Mohamed D, Hochkirch U, Thomale J and Linscheid MW: Structures of oxaliplatin-oligonucleotide adducts from DNA. J Mass Spectrom 47: 1282-1293, 2012.

5. Virag P, Perde-Schrepler M, Fischer-Fodor E, Tatomir C, Dorneanu SA, Cernea VI and Irimie A: Superior cytotoxicity and DNA cross-link induction by oxaliplatin versus cisplatin at lower cellular uptake in colorectal cancer cell lines. Anticancer Drugs 23: 1032-1038, 2012.

6. Saltz LB, Clarke S, Diaz-Rubio E, Scheithauer W, Figer A, Wong R, Koski S, Lichinitser M, Yang TS, Rivera F, et al: Bevacizumab in combination with oxaliplatin-based chemotherapy as first-line therapy in metastatic colorectal cancer: A randomized phase III study. J Clin Oncol 26: 2013-2019, 2008.

7. Douillard JY, Siena S, Cassidy J, Tabernero J, Burkes R, Barugel M, Humblet Y, Bodoky G, Cunningham D, Jassem J, et al: Randomized, phase III trial of panitumumab with infusional fluorouracil, leucovorin, and oxaliplatin (FOLFOX4) versus FOLFOX4 alone as first-line treatment in patients with previously untreated metastatic colorectal cancer: The PRIME study. J Clin Oncol 28: 4697-4705, 2010.

8. Bokemeyer C, Bondarenko I, Makhson A, Hartmann JT, Aparicio J, de Braud F, Donea S, Ludwig H, Schuch G, Stroh C, et al: Fluorouracil, leucovorin, and oxaliplatin with and without cetuximab in the first-line treatment of metastatic colorectal cancer. J Clin Oncol 27: 663-671, 2009.

9. Zheng N, Schulman BA, Song L, Miller JJ, Jeffrey PD, Wang P, Chu C, Koepp DM, Elledge SJ, Pagano M, et al: Structure of the Cul1-Rbx1-Skp1-F boxSkp2 SCF ubiquitin ligase complex. Nature 416: 703-709, 2002.

10. Soucy TA, Smith PG, Milhollen MA, Berger AJ, Gavin JM, Adhikari S, Brownell JE, Burke KE, Cardin DP, Critchley S, et al: An inhibitor of NEDD8-activating enzyme as a new approach to treat cancer. Nature 458: 732-736, 2009.

11. Petroski MD and Deshaies RJ: Function and regulation of cullin-RING ubiquitin ligases. Nat Rev Mol Cell Biol 6: 9-20, 2005.

12. Petroski MD: Mechanism-based neddylation inhibitor. Chem Biol 17: 6-8, 2010

13. Soucy TA, Dick LR, Smith PG, Milhollen MA and Brownell JE: The NEDD8 conjugation pathway and its relevance in cancer biology and therapy. Genes Cancer 1: 708-716, 2010

14. Brownell JE, Sintchak MD, Gavin JM, Liao H, Bruzzese FJ, Bump NJ, Soucy TA, Milhollen MA, Yang X, Burkhardt AL, et al: Substrate-assisted inhibition of ubiquitin-like protein-activating enzymes: The NEDD8 E1 inhibitor MLN4924 forms a NEDD8-AMP mimetic in situ. Mol Cell 37: 102-111, 2010.

15. Liao H, Liu XJ, Blank JL, Bouck DC, Bernard H, Garcia K and Lightcap ES: Quantitative proteomic analysis of cellular protein modulation upon inhibition of the NEDD8-activating enzyme by MLN4924. Mol Cell Proteomics 10: M111.009183, 2011.
16. Bennett EJ, Rush J, Gygi SP and Harper JW: Dynamics of cullin-RING ubiquitin ligase network revealed by systematic quantitative proteomics. Cell 143: 951-965, 2010.

17. Milhollen MA, Traore T, Adams-Duffy J, Thomas MP, Berger AJ, Dang L, Dick LR, Garnsey JJ, Koenig E, Langston SP, et al: MLN4924, a NEDD8-activating enzyme inhibitor, is active in diffuse large B-cell lymphoma models: Rationale for treatment of NF-\{kappa\}B-dependent lymphoma. Blood 116: 1515-1523, 2010.

18. Jia L, Li H and Sun Y: Induction of p21-dependent senescence by an NAE inhibitor, MLN4924, as a mechanism of growth suppression. Neoplasia 13: 561-569, 2011.

19. Swords RT, Kelly KR, Smith PG, Garnsey JJ, Mahalingam D, Medina E, Oberheu K, Padmanabhan S, O'Dwyer M, Nawrocki ST, et al: Inhibition of NEDD8-activating enzyme: A novel approach for the treatment of acute myeloid leukemia. Blood 115: 3796-3800, 2010

20. Lin JJ, Milhollen MA, Smith PG, Narayanan U and Dutta A NEDD8-targeting drug MLN4924 elicits DNA rereplication by stabilizing Cdt1 in $\mathrm{S}$ phase, triggering checkpoint activation, apoptosis, and senescence in cancer cells. Cancer Res 70 10310-10320, 2010.

21. Milhollen MA, Narayanan U, Soucy TA, Veiby PO, Smith PG and Amidon B: Inhibition of NEDD8-activating enzyme induces rereplication and apoptosis in human tumor cells consistent with deregulating CDT1 turnover. Cancer Res 71: 3042-3051, 2011.

22. Wei D, Li H, Yu J, Sebolt JT, Zhao L, Lawrence TS, Smith PG, Morgan MA and Sun Y: Radiosensitization of human pancreatic cancer cells by MLN4924, an investigational NEDD8-activating enzyme inhibitor. Cancer Res 72: 282-293, 2012.

23. Luo Z, Yu G, Lee HW, Li L, Wang L, Yang D, Pan Y, Ding C, Qian J, Wu L, et al: The Nedd8-activating enzyme inhibitor MLN4924 induces autophagy and apoptosis to suppress liver cancer cell growth. Cancer Res 72: 3360-3371, 2012.

24. Woynarowski JM, Faivre S, Herzig MC, Arnett B, Chapman WG Trevino AV, Raymond E, Chaney SG, Vaisman A, Varchenko M and Juniewicz PE: Oxaliplatin-induced damage of cellular DNA Mol Pharmacol 58: 920-927, 2000.

25. Nawrocki ST, Kelly KR, Smith PG, Espitia CM, Possemato A, Beausoleil SA, Milhollen M, Blakemore S, Thomas M, Berger A and Carew JS: Disrupting protein NEDDylation with MLN4924 is a novel strategy to target cisplatin resistance in ovarian cancer. Clin Cancer Res 19: 3577-3590, 2013.

26. Rogakou EP, Nieves-Neira W, Boon C, Pommier Y and Bonner WM: Initiation of DNA fragmentation during apoptosis induces phosphorylation of H2AX histone at serine 139. J Biol Chem 275: 9390-9395, 2000.

27. Matsuoka S, Rotman G, Ogawa A, Shiloh Y, Tamai K and Elledge SJ: Ataxia telangiectasia-mutated phosphorylates Chk2 in vivo and in vitro. Proc Natl Acad Sci USA 97: 10389-10394, 2000.

28. Bunz F, Dutriaux A, Lengauer C, Waldman T, Zhou S, Brown JP, Sedivy JM, Kinzler KW and Vogelstein B: Requirement for p53 and p21 to sustain G2 arrest after DNA damage. Science 282: 1497-1501, 1998

29. Hirao A, Kong YY, Matsuoka S, Wakeham A, Ruland J, Yoshida H, Liu D, Elledge SJ and Mak TW: DNA damage-induced activation of $\mathrm{p} 53$ by the checkpoint kinase Chk2. Science 287 : 1824-1827, 2000

30. Rainey MD, Black EJ, Zachos G and Gillespie DA: Chk2 is required for optimal mitotic delay in response to irradiation-induced DNA damage incurred in G2 phase. Oncogene 27: 896-906, 2008

31. Kee Y, Huang M, Chang S, Moreau LA, Park E, Smith PG and D'Andrea AD: Inhibition of the Nedd8 system sensitizes cells to DNA interstrand cross-linking agents. Mol Cancer Res 10 : 369-377, 2012.

32. Fink D, Nebel S, Aebi S, Zheng H, Cenni B, Nehmé A, Christen RD and Howell SB: The role of DNA mismatch repair in platinum drug resistance. Cancer Res 56: 4881-4886, 1996. 